Article

\title{
On the Number of Periodic Orbits to Odd Order Differential Delay Systems
}

\author{
Weigao $\mathrm{Ge}^{\dagger}$ and $\mathrm{Lin} \mathrm{Li}^{*},+$
}

School of Mathematics and Statistics, Beijing Institute of Technology, Beijing 100081, China; gew@bit.edu.cn

* Correspondence: li_boy_ok@126.com

t These authors contributed equally to this work.

Received: 14 July 2020; Accepted: 24 September 2020; Published: 9 October 2020

\begin{abstract}
In this paper, we study the periodic orbits of a type of odd order differential delay system with $2 k-1$ lags via the $S^{1}$ index theory and the variational method. This type of system has not been studied by others. Our results provide a new and more accurate method for counting the number of periodic orbits.
\end{abstract}

Keywords: odd order differential delay system; periodic orbits; critical point theory; variational method

\section{Introduction}

The delay differential equations have useful applications in various fields such as age-structured population growth, life sciences, control theory, and any model involving responses with non-zero delays [1,2].

The problem of periodic solutions for multi-delay differential equations started from the research work of Kaplan and Yorke [3] in 1974. Consider the following differential delay equation

$$
x^{\prime}(t)=-\sum_{i=1}^{n} f(x(t-i))
$$

in which $f(-x)=-f(x), x f(x)>0, x \neq 0$. They proved the existence of the periodic solutions of (1) when $n=1$ and $n=2$, respectively. In the subsequent literatures [4-17], the number of $2(n+1)$-periodic solutions of Equation (1) was studied by using the results of Nussbaum [18]. In 1998, $\mathrm{Li}$ and $\mathrm{He}$ [15] made some researches for (1) by use of the theory of symmetric group. Especially, in 2006, Fei used the variational method to study the number of periodic solutions of Equation (1). He studied the cases of $n=2 k-1$ and $n=2 k$ respectively in literature [4,5] since the specific research methods and details vary greatly. Almost at the same time, Guo and Yu [13] applied critical point theories to study the multiplicity of the 4-periodic solutions for (1) when $n=1$. After then they [12] studied the same problem for (1) when $n=2 k-1, k \geq 1$, which is an extension of Fei's article [4] but the proof of theorem is more difficult and more complicated. In addition, all of these researches are about first order differential equations and did not give the precise counting method for the number of periodic solutions.

The goal of this paper is to study the periodic orbits to a type of odd order differential delay system in the form

$$
\left\{\begin{array}{l}
x^{(2 m+1)}(t)=-\sum_{p=1}^{2 k-1} \nabla F(x(t-p)), \text { a.e. } t \in[0,4 k] \\
x(t)-x(t-4 k)=0 .
\end{array}\right.
$$


where

$$
x \in \mathbb{R}^{N}, F \in C^{1}\left(\mathbb{R}^{N}, \mathbb{R}\right), \nabla F(-x)=-\nabla F(x), F(0)=0
$$

and there are real symmetric matrices $A_{0}, A_{\infty} \in \mathbb{R}^{N \times N}$ such that

$$
\nabla F(x)=A_{0} x+\circ(|x|),|x| \rightarrow 0, \quad \nabla F(x)=A_{\infty} x+\circ(|x|),|x| \rightarrow \infty .
$$

The method applied in this paper is the $S^{1}$ index theory and the variational approach [19-21]. The variation structure we built in this paper is simpler compared with [13]. In addition, the counting method for the number of periodic orbits in our results only depends on the eigenvalues of $A_{\infty}$ and $A_{0}$. So it is more precise and easier to examine.

To facilitate the following discussion, we suppose that

$$
\alpha_{1} \leq \alpha_{2} \leq \cdots \leq \alpha_{N}, \beta_{1} \leq \beta_{2} \leq \cdots \leq \beta_{N}
$$

are respectively the eigenvalues of $A_{0}$ and $A_{\infty}$. Their corresponding unit eigenvectors are respectively $u_{1}, u_{2}, \cdots, u_{N} ; v_{1}, v_{2} \cdots, v_{N}$. At the same time, the one-dimensional space corresponding to each eigenvector is denoted as

$$
U_{j}=\operatorname{span}\left\{u_{j}\right\}, \quad V_{j}=\operatorname{span}\left\{v_{j}\right\}, \quad j=1,2, \cdots, N .
$$

\section{Space $X$ and Functional $\Phi$}

Consider the $4 k$-periodic orbits of (2), and suppose

$$
x(t-2 k)=-x(t), k \geq 1 .
$$

Let

$$
\begin{gathered}
\widehat{X}=\left\{x \in L^{2}: x(t-2 k)=-x(t)\right\}=\left\{\sum_{j=0}^{\infty}\left(a_{j} \cos \frac{(2 j+1) \pi t}{2 k}+b_{j} \sin \frac{(2 j+1) \pi t}{2 k}\right): a_{j}, b_{j} \in \mathbb{R}^{N}\right\}, \\
X=c l\left\{\sum_{j=0}^{\infty}\left(a_{j} \cos \frac{(2 j+1) \pi t}{2 k}+b_{j} \sin \frac{(2 j+1) \pi t}{2 k}\right): a_{j}, b_{j} \in \mathbb{R}, \sum_{j=0}^{\infty}(2 j+1)^{2 m+1}\left(a_{j}^{2}+b_{j}^{2}\right)<\infty\right\} \subset \widehat{X},
\end{gathered}
$$

and define $P: X \rightarrow L^{2}$ by

$$
\begin{aligned}
P^{2 m+1} x(t) & =P^{2 m+1}\left(\sum_{j=0}^{\infty}\left(a_{j} \cos \frac{(2 j+1) \pi t}{2 k}+b_{j} \sin \frac{(2 j+1) \pi t}{2 k}\right)\right) \\
& =\sum_{j=0}^{\infty}(2 j+1)^{2 m+1}\left(a_{j} \cos \frac{(2 j+1) \pi t}{2 k}+b_{j} \sin \frac{(2 j+1) \pi t}{2 k}\right) .
\end{aligned}
$$

Let

$$
P^{-(2 m+1)} x(t)=\sum_{j=0}^{\infty} \frac{1}{(2 j+1)^{2 m+1}}\left(a_{j} \cos \frac{(2 i+1) \pi t}{2 k}+b_{j} \sin \frac{(2 j+1) \pi t}{2 k}\right) .
$$

For $x \in X$, define

$$
\begin{gathered}
\langle x, y\rangle=\int_{0}^{4 k}\left(P^{2 m+1} x(t), y(t)\right) d t,\|x\|=\sqrt{\langle x, x\rangle}, \\
\langle x, y\rangle_{0}=\int_{0}^{4 k}(x(t), y(t)) d t,\|x\|_{0}=\sqrt{\langle x, x\rangle_{0}} .
\end{gathered}
$$


Therefore, $(X,\|\cdot\|)$ is an $H^{m+\frac{1}{2}}\left([0,4 k], \mathbb{R}^{N}\right)$ space.

In order to facilitate subsequent calculations, we make a more detailed division as follows,

$$
\begin{gathered}
X(j)=\left\{x(t)=a_{j} \cos \frac{(2 j+1) \pi t}{2 k}+b_{j} \sin \frac{(2 j+1) \pi t}{2 k}: a_{j}, b_{j} \in \mathbb{R}^{N}\right\}, \\
X_{0, i}(j)=c l\left\{x(t)=a_{j} \cos \frac{(2 j+1) \pi t}{2 k}+b_{j} \sin \frac{(2 j+1) \pi t}{2 k}: a_{j}, b_{j} \in U_{i}\right\}, \\
X_{\infty, i}(j)=c l\left\{x(t)=a_{j} \cos \frac{(2 j+1) \pi t}{2 k}+b_{j} \sin \frac{(2 j+1) \pi t}{2 k}: a_{j}, b_{j} \in V_{i}\right\},
\end{gathered}
$$

then we have $X(j)=\sum_{i=1}^{N} X_{0, i}(j)=\sum_{i=1}^{N} X_{\infty, i}(j)$, and let

$$
X_{0, i}=\bigoplus_{j=0}^{\infty} X_{0, i}(j), \quad X_{\infty, i}=\bigoplus_{j=0}^{\infty} X_{\infty, i}(j), \quad X=\underset{1 \leq i \leq N}{\oplus} X_{0, i}=\underset{1 \leq i \leq N}{\bigoplus} X_{\infty, i} .
$$

It is easy to see that, $\forall x \in X(j)$,

$$
\|x\|^{2}=(2 j+1)^{(2 m+1)}\|x\|_{0}^{2} .
$$

Then, we can get

$$
\int_{0}^{4 k}\left(A_{0} x(t), x(t)\right) d t=\alpha_{i}\|x\|_{0}^{2}, \forall x \in X_{0, i}(j) \subset X(j),
$$

and

$$
\int_{0}^{4 k}\left(A_{\infty} x(t), x(t)\right) d t=\beta_{i}\|x\|_{0}^{2}, \forall x \in X_{\infty, i}(j) \subset X(j) .
$$

For the system (2), define the following functional

$$
\Phi(x)=\frac{1}{2}\langle L x, x\rangle+\int_{0}^{4 k} F(x(t)) d t
$$

where

$$
\begin{gathered}
L x=P^{-(2 m+1)} \sum_{j=1}^{2 k-1}(-1)^{j} x^{(2 m+1)}(t-j) . \\
\text { If } x_{j}(t)=a_{j} \cos \frac{(2 j+1) \pi t}{2 k}+b_{j} \sin \frac{(2 j+1) \pi t}{2 k} \in X(j) \text {, we have } \\
L x=(-1)^{m+1}\left(\frac{\pi}{2 k}\right)^{2 m+1}\left(\sum_{j=0}^{\infty} x_{j} \tan \frac{(2 j+1) \pi}{4 k}\right) .
\end{gathered}
$$

Obviously $\left.L\right|_{X(j)}: X(j) \rightarrow X(j)$ is invertible.

According to Theorem 1.4 in [21], the differential of functional $\Phi$ is

$$
P^{-(2 m+1)} \Phi^{\prime}(x)=L x+\Psi(x)
$$

where $\Psi(x)=P^{-(2 m+1)} \nabla F(x)$. It is easy to prove that $\Psi:\left(X,\|x\|^{2}\right) \rightarrow\left(X,\|x\|_{0}^{2}\right)$ is compact. 


\section{Partition of Space $X$ and Symbols}

From (10), we have that if

$$
x(t)=\sum_{j=0}^{\infty}\left(a_{j} \cos \frac{(2 j+1) \pi t}{2 k}+b_{j} \sin \frac{(2 j+1) \pi t}{2 k}\right) \in X(j),
$$

then

$$
\begin{aligned}
\langle L x, x\rangle & =(-1)^{m+1}\left(\frac{\pi}{2 k}\right)^{2 m+1}(2 j+1)^{2 m+1} \tan \frac{(2 j+1) \pi}{4 k}\|x\|_{0}^{2} \\
& =(-1)^{m+1}\left(\frac{\pi}{2 k}\right)^{2 m+1} \tan \frac{(2 j+1) \pi}{4 k}\|x\|^{2} .
\end{aligned}
$$

Thereby, $\forall x \in X_{0, i}(2 l k+j) \subset X(2 l k+j), j=0,1,2, \cdots, 2 k-1$, we have

$$
\begin{aligned}
& \left\langle\left(L+P^{-(2 m+1)} A_{0}\right) x, x\right\rangle \\
= & {\left[(-1)^{m+1}\left(\frac{\pi}{2 k}\right)^{2 m+1}(4 l k+2 j+1)^{2 m+1} \tan \frac{(2 j+1) \pi}{4 k}+\alpha_{i}\right]\|x\|_{0}^{2} . }
\end{aligned}
$$

Similarly, $\forall x \in X_{\infty, i}(2 l k+j) \subset X(2 l k+j), j=0,1,2, \cdots, 2 k-1$, we have

$$
\begin{aligned}
& \left\langle\left(L+P^{-(2 m+1)} A_{\infty}\right) x, x\right\rangle \\
= & {\left[(-1)^{m+1}\left(\frac{\pi}{2 k}\right)^{2 m+1}(4 l k+2 j+1)^{2 m+1} \tan \frac{(2 j+1) \pi}{4 k}+\beta_{i}\right]\|x\|_{0}^{2} . }
\end{aligned}
$$

Hence, define

$$
\left\{\begin{array}{l}
X_{0, i}^{+}(2 l k+j)=\left\{X_{0, i}(2 l k+j):\left[(-1)^{m+1}\left(\frac{\pi}{2 k}\right)^{2 m+1}(4 l k+2 j+1)^{2 m+1} \tan \frac{(2 j+1) \pi}{4 k}+\alpha_{i}\right]>0\right\}, \\
X_{0, i}^{0}(2 l k+j)=\left\{X_{0, i}(2 l k+j):(-1)^{m+1}\left(\frac{(4 l k+2 j+1) \pi}{2 k}\right)^{2 m+1} \tan \frac{(2 j+1) \pi}{4 k}=-\alpha_{i}\right\}, \\
X_{0, i}^{-}(2 l k+j)=\left\{X_{0, i}(2 l k+j):\left[(-1)^{m+1}\left(\frac{\pi}{2 k}\right)^{2 m+1}(4 l k+2 j+1)^{2 m+1} \tan \frac{(2 j+1) \pi}{4 k}+\alpha_{i}\right]<0\right\}, \\
X_{\infty, i}^{+}(2 l k+j)=\left\{X_{\infty, i}(2 l k+j):\left[(-1)^{m+1}\left(\frac{\pi}{2 k}\right)^{2 m+1}(4 l k+2 j+1)^{2 m+1} \tan \frac{(2 j+1) \pi}{4 k}+\beta_{i}\right]>0\right\}, \\
X_{\infty, i}^{0}(2 l k+j)=\left\{X_{\infty, i}(2 l k+j):(-1)^{m+1}\left(\frac{(4 l k+2 j+1) \pi}{2 k}\right)^{2 m+1} \tan \frac{(2 j+1) \pi}{4 k}=-\beta_{i}\right\}, \\
X_{\infty, i}^{-}(2 l k+j)=\left\{X_{\infty, i}(2 l k+j):\left[(-1)^{m+1}\left(\frac{\pi}{2 k}\right)^{2 m+1}(4 l k+2 j+1)^{2 m+1} \tan \frac{(2 j+1) \pi}{4 k}+\beta_{i}\right]<0\right\} .
\end{array}\right.
$$

Then, we have

$$
\begin{aligned}
& X_{\infty, i}^{+}=\sum_{j=0}^{2 k-1} \sum_{l=0}^{\infty} X_{\infty, i}^{+}(2 l k+j), X_{\infty, i}^{0}=\sum_{j=0}^{2 k-1} \sum_{l=0}^{\infty} X_{\infty, i}^{0}(2 l k+j), X_{\infty, i}^{-}=\sum_{j=0}^{2 k-1} \sum_{l=0}^{\infty} X_{\infty, i}^{-}(2 l k+j), \\
& X_{0, i}^{+}=\sum_{j=0}^{2 k-1} \sum_{l=0}^{\infty} X_{0, i}^{+}(2 l k+j), X_{\infty, i}^{0}=\sum_{j=0}^{2 k-1} \sum_{l=0}^{\infty} X_{0, i}^{0}(2 l k+j), X_{0, i}^{-}=\sum_{j=0}^{2 k-1} \sum_{l=0}^{\infty} X_{0, i}^{-}(2 l k+j) .
\end{aligned}
$$

Thus,

$$
\begin{array}{ll}
X_{0}^{+}(2 l k+j)=\bigoplus_{i=1}^{N} X_{0, i}^{+}, & X_{\infty}^{+}(2 l k+j)=\bigoplus_{i=1}^{N} X_{\infty, i}^{+}, \\
X_{0}^{0}(2 l k+j)=\bigoplus_{i=1}^{N} X_{0, i}^{0}, & X_{\infty}^{0}(2 l k+j)=\bigoplus_{i=1}^{N} X_{\infty, i}^{0}, \\
X_{0}^{-}(2 l k+j)=\bigoplus_{i=1}^{N} X_{0, i^{\prime}}^{-} & X_{\infty}^{-}(2 l k+j)=\bigoplus_{i=1}^{N} X_{\infty, i}^{-} .
\end{array}
$$


It is easy to see that $\left|(-1)^{m+1}\left(\frac{(2 j+1) \pi}{2 k}\right)^{2 m+1} \tan \frac{(2 j+1) \pi}{4 k}\right| \rightarrow \infty$ as $j \rightarrow \infty$, then we can get that $\operatorname{dim} X_{\infty}^{0}<\infty$ and $\operatorname{dim} X_{0}^{0}<\infty$.

\section{Lemmas}

Let $X$ be a Hilbert space, $L: X \rightarrow X$ be a linear operator, and $\Phi: X \rightarrow R$ be a differentiable functional.

Lemma 1 ([4], Lemma 2.4). Assume that there are two closed $s^{1}$-invariant linear subspaces, $X^{+}$and $X^{-}$, and $r>0$ such that

(a) $X^{+} \cup X^{-}$is closed and of finite codimensions in $X$,

(b) $\widehat{L}\left(X^{-}\right) \subset X^{-}, \widehat{L}=L+P^{-1} A_{0}$ or $\widehat{L}=L+P^{-1} A_{\infty}$,

(c) there exists $c_{0} \in R$ such that

$$
\inf _{x \in X^{+}} \Phi(x) \geq c_{0}
$$

(d) there is $c_{\infty} \in R$ such that

$$
\Phi(x) \leq c_{\infty}<\Phi(0)=0, \forall x \in X^{-} \cap S_{r}=\left\{x \in X^{-}:\|x\|=r\right\}
$$

(e) $\Phi$ satisfies $(P . S)_{c}$-condition, $c_{0}<c<c_{\infty}$, i.e., every sequence $\left\{x_{n}\right\} \subseteq X$ with $\Phi\left(x_{n}\right) \rightarrow c$ and $\Phi^{\prime}\left(x_{n}\right) \rightarrow 0$ possesses a convergent subsequence.

Then $\Phi$ has at least $\frac{1}{2}\left[\operatorname{dim}\left(X^{+} \cap X^{-}\right)-\operatorname{codim}_{X}\left(X^{+} \cup X^{-}\right)\right]$generally different critical orbits in $\Phi^{-1}\left(\left[c_{0}, c_{\infty}\right]\right)$ if $\left[\operatorname{dim}\left(X^{+} \cap X^{-}\right)-\operatorname{codim}_{X}\left(X^{+} \cup X^{-}\right)\right]>0$

In addition, we make the following assumptions for convenience,

$\left(S_{1}\right) f$ satisfies (3) and (4),

$\left(S_{2}\right)\left[F(x)-\frac{1}{2}\left(A_{\infty} x, x\right)\right] \rightarrow \infty, \quad|x| \rightarrow \infty$.

Lemma 2. Under the assumptions of $\left(S_{1}\right)$ and $\left(S_{2}\right)$, there exists $\sigma>0$ such that

$$
\left\langle\left(L+P^{-(2 m+1)} A_{\infty}\right) x, x\right\rangle>\sigma\|x\|^{2}, \quad x \in X_{\infty}^{+} \text {and }\left\langle\left(L+P^{-(2 m+1)} A_{\infty}\right) x, x\right\rangle<-\sigma\|x\|^{2}, x \in X_{\infty}^{-} .
$$

Proof of Lemma 2. Let $\Gamma_{i}: X \rightarrow X_{i}, i \in\{1,2, \cdots, N\}$ be the orthogonal projection, then $i d=\sum_{i=1}^{N} \Gamma_{i}$. Assuming that $x \in X_{\infty}^{+}$, then for $x_{i}=\Gamma_{i} x \in X_{\infty, i}^{+}$, we can get

$$
x_{i}(t)=\sum_{l=0}^{\infty} \sum_{j=0}^{2 k-1} x_{\infty, i}^{+}(2 l k+j)
$$

Then from (12), we can get

$$
\begin{aligned}
& \left\langle\left(L+P^{-(2 m+1)} A_{\infty}\right) x_{i}, x_{i}\right\rangle \\
= & \sum_{l=0}^{\infty} \sum_{j=0}^{2 k-1}\left\langle\left(L+P^{-(2 m+1)} A_{\infty, i}\right) x_{\infty, i}^{+}(2 l k+j), x_{\infty, i}^{+}(2 l k+j)\right\rangle \\
= & \sum_{l=0}^{\infty} \sum_{j=0}^{2 k-1}\left[(-1)^{m+1}\left(\frac{\pi}{2 k}\right)^{2 m+1}(4 l k+2 j+1)^{2 m+1} \tan \frac{(2 j+1) \pi}{4 k}+\beta_{i}\right]\left\|x_{\infty, i}^{+}(2 l k+j)\right\|_{0}^{2} \\
= & \sum_{l=0}^{\infty} \sum_{j=0}^{2 k-1}\left[(-1)^{m+1}\left(\frac{\pi}{2 k}\right)^{2 m+1} \tan \frac{(2 j+1) \pi}{4 k}+\frac{\beta_{i}}{(4 l k+2 j+1)^{2 m+1}}\right]\left\|x_{\infty, i}^{+}(2 l k+j)\right\|^{2}
\end{aligned}
$$


For convenience, let

$$
h_{i, j}(l)=(-1)^{m+1}\left(\frac{\pi}{2 k}\right)^{2 m+1} \tan \frac{(2 j+1) \pi}{4 k}+\frac{\beta_{i}}{(4 l k+2 j+1)^{2 m+1}},
$$

then

$$
\begin{aligned}
& \left\langle\left(L+P^{-(2 m+1)} A_{\infty}\right) x_{i}, x_{i}\right\rangle \\
= & \sum_{l=0}^{\infty} \sum_{j=0}^{2 k-1} h_{i, j}(l)\left\|x_{\infty, i}^{+}(2 l k+j)\right\|^{2}
\end{aligned}
$$

in which $h_{i, j}(l)>0$ as $x_{i} \in X_{\infty, i}^{+}$.

Furthermore, let $\delta=(-1)^{m+1}\left(\frac{\pi}{2 k}\right)^{2 m+1} \tan \frac{(2 j+1) \pi}{4 k}$ and $N_{i, j}^{+}=\left\{l: h_{i, j}(l)>0\right\}$. If $\delta<0$, then $\lim _{l \rightarrow \infty} h_{i, j}(l)=\delta<0$. It follows that $N_{i, j}^{+}$is a finite set and then there exists $l_{0} \in N_{i, j}^{+}$, such that $h_{i, j}\left(l_{0}\right)=\min \left\{h_{i, j}(l): l \in N_{i, j}^{+}\right\}>0$. In this case, let $\sigma_{i, j,\left(1^{\prime}\right)}=h_{i, j}\left(l_{0}\right)$.

On the other hand, if $\delta>0$, then there exists $L>0$, such that $h_{i, j}(l)>\frac{\delta}{2}$ when $l>L$. Therefore, $\exists \hat{l} \in N_{i, j}^{+} \cap\{0,1, \cdots, L\}$, such that

$$
h_{i, j}(\hat{l})=\min \left\{h_{i, j}(l): l \in N_{i, j}^{+} \bigcap\{0,1, \cdots, L\}\right\}>0 .
$$

In this case, let $\sigma_{i, j,\left(1^{\prime \prime}\right)}=\min \left\{h_{i, j}(\hat{l}), \frac{\delta}{2}\right\}$. Then let $\sigma_{i, j,(1)}=\min \left\{\sigma_{i, j,\left(1^{\prime}\right)}, \sigma_{i, j,\left(1^{\prime \prime}\right)}\right\}$, one has $h_{i, j}(l)>\sigma_{i, j,(1)}>0$.

Similarly, if $x_{i}(t)=\sum_{l=0}^{\infty} \sum_{j=0}^{2 k-1} x_{\infty, i}^{-}(2 l k+j) \in X_{\infty, i}^{-}$one has $\exists \sigma_{i, j,(2)}>0$, such that

$$
h_{i, j}(l)<-\sigma_{i, j,(2)}<0 .
$$

Then let $\sigma_{i, j}=\min \left\{\sigma_{i, j,(1)}, \sigma_{i, j,(2)}\right\}>0$, we can get that

$$
\left\langle\left(L+P^{-(2 m+1)} A_{\infty} x_{i}, x_{i}\right\rangle=\sum_{l=0}^{\infty} \sum_{j=0}^{2 k-1} h_{i, j}(l)\left\|x_{\infty, i}^{+}(2 l k+j)\right\|^{2}>\sigma_{i, j} \sum_{l=0}^{\infty} \sum_{j=0}^{2 k-1}\left\|x_{\infty, i}^{+}(2 l k+j)\right\|^{2}\right.
$$

when $x_{i} \in X_{\infty, i}^{+}$, and

$$
\left\langle\left(L+P^{-(2 m+1)} A_{\infty} x_{i}, x_{i}\right\rangle=\sum_{l=0}^{\infty} \sum_{j=0}^{2 k-1} h_{i, j}(l)\left\|x_{\infty, i}^{-}(2 l k+j)\right\|^{2}<-\sigma_{i, j} \sum_{l=0}^{\infty} \sum_{j=0}^{2 k-1}\left\|x_{\infty, i}^{-}(2 l k+j)\right\|^{2}\right.
$$

when $x_{i} \in X_{\infty, i}^{-}$.

Then let $\sigma=\min _{\substack{0 \leq j \leq 2 k-1 \\ 1 \leq i \leq N}} \sigma_{i, j}>0$. The inequalities in (14) are proved.

Lemma 3. Under the assumptions of $\left(S_{1}\right)$ and $\left(S_{2}\right)$, the functional $\Phi$ defined by (9) satisfies (P.S)-condition.

Proof of Lemma 3. Let $\Gamma_{i}: X \rightarrow X_{\infty, i}$ be the orthogonal projections, then $x_{n, i}=\Gamma_{i} x_{n} \in X_{i}, x_{n}=\sum_{i=1}^{N} x_{n, i}$.

Assume that $\left\{x_{n}\right\} \subset X$ is a subsequence such that $\Phi^{\prime}\left(x_{n}\right) \rightarrow 0$ and $\Phi\left(x_{n}\right)$ is bounded. Then we can get that, $\forall i \in\{1,2, \cdots, N\}, \Phi^{\prime}\left(x_{n, i}\right) \rightarrow 0$ and $\Phi\left(x_{n, i}\right)$ is bounded. Let $x_{n, i}^{+}=x_{n, i} \cap X_{\infty, i}^{+}, x_{n, i}^{-}=$ 
$x_{n, i} \cap X_{\infty, i^{\prime}}^{-} \quad x_{n, i}^{0}=x_{n, i} \cap X_{\infty, i}^{0}$. Similarly, $\Phi^{\prime}\left(x_{n, i}^{+}\right), \Phi^{\prime}\left(x_{n, i}^{-}\right), \Phi^{\prime}\left(x_{n, i}^{0}\right) \rightarrow 0$ when $n \rightarrow \infty$ and $\Phi\left(x_{n, i}^{+}\right), \Phi\left(x_{n, i}^{-}\right), \Phi\left(x_{n, i}^{0}\right)$ are bounded. According to (4), we can get that

$$
\left|\left\langle P^{-(2 m+1)}\left(\nabla F\left(x_{n, i}^{+}\right)-A_{\infty} x_{n, i}^{+}\right), x_{n, i}^{+}\right\rangle\right|<\frac{\sigma}{2}\left\|x_{n, i}^{+}\right\|^{2}+M,
$$

for some $M>0$. Thus, from

$$
\left\langle P^{-(2 m+1)} \Phi^{\prime}\left(x_{n, i}^{+}\right), x_{n, i}^{+}\right\rangle=\left\langle L x_{n}+P^{-(2 m+1)} \nabla F\left(x_{n, i}^{+}\right), x_{n, i}^{+}\right\rangle \rightarrow 0,
$$

and (14), we can get

$$
\begin{aligned}
& \left\langle P^{-(2 m+1)} \Phi^{\prime}\left(x_{n, i}^{+}\right), x_{n, i}^{+}\right\rangle \\
\geq & \left|\left\langle\left(L+P^{-(2 m+1)} A_{\infty}\right) x_{n, i}^{+}, x_{n, i}^{+}\right\rangle\right|-\left|\left\langle P^{-(2 m+1)}\left(\nabla F\left(x_{n, i}^{+}\right)-A_{\infty} x_{n, i}^{+}\right), x_{n, i}^{+}\right\rangle\right| \\
\geq & \frac{\sigma}{2}|| x_{n, i}^{+} \|^{2}-M,
\end{aligned}
$$

which implies the boundedness of $\left\|x_{n, i}^{+}\right\|$. Then we can get the boundedness of $\left\|x_{n}^{+}\right\|$. Similarly, we have the boundedness of $\left\|x_{n}^{-}\right\|$. At the same time, $\left\|x_{n}^{0}\right\|$ is bounded since $X_{n}^{0}$ is finite-dimensional. Therefore, $\left\|x_{n}\right\|$ is bounded. It follows from (11) that

$$
P^{-(2 m+1)} \Phi^{\prime}\left(x_{n}\right)=L x_{n}+K\left(x_{n}\right) .
$$

Considering the compactness of operator $K$ and the boundedness of $x_{n}$, we can get that $K\left(x_{n}\right) \rightarrow u$. Then, we have that

$$
L x_{n} \rightarrow-u
$$

as $\Phi^{\prime}\left(x_{n}\right) \rightarrow 0$. Therefore,

$$
x_{n} \rightarrow-L^{-1} u,
$$

which implies (P.S)-condition.

Lemma 4. If $x$ is a critical point of $\Phi$, then the orbit corresponding to $x$ is a periodic orbit to the system (2).

Proof of Lemma 4. Suppose $x$ is a critical point of $\Phi$ given by (9). Then $x(t)$ should satisfy

$$
-\sum_{j=1}^{2 k-1}(-1)^{j+1} x^{(2 m+1)}(t-j)+\nabla F(x(t))=0 \text {, a.e. } t \in[0,4 k] .
$$

Consequently,

$$
\begin{gathered}
-\sum_{j=1}^{2 k-1}(-1)^{j+1} x^{(2 m+1)}(t-j-1)+\nabla F(x(t-1))=0, \\
-\sum_{j=1}^{2 k-1}(-1)^{j+1} x^{(2 m+1)}(t-j-2)+\nabla F(x(t-2))=0, \\
-\sum_{j=1}^{2 k-1}(-1)^{j+1} x^{(2 m+1)}(t-j-3)+\nabla F(x(t-3))=0, \\
\vdots \\
-\sum_{j=0}^{2 k-1}(-1)^{j+1} x^{(2 m+1)}(t-j-(2 k-1))+\nabla F(x(t-(2 k-1)))=0 .
\end{gathered}
$$


Calculating $(15 a)+(15 b)+(15 c)+\cdots+(15(2 k-1))$, we have

$$
x^{(2 m+1)}(t)+\sum_{j=1}^{2 k-1} \nabla F(x(t-j))=0, \text { a.e. } t \in[0,4 k],
$$

namely,

$$
x^{(2 m+1)}(t)=-\sum_{j=1}^{2 k-1} \nabla F(x(t-j)), \text { a.e. } t \in[0,4 k],
$$

hence, the orbit corresponding to $\mathrm{x}$ is a periodic orbit of (2).

\section{Main Results}

From the definition of (13), we can get that there exists $d>0$, such that

$$
\left\{\begin{array}{l}
X_{\infty, i}^{+}(2 l k+j)=X_{\infty, i}(2 l k+j), \\
X_{0, i}^{-}(2 l k+j)=\phi \\
X_{\infty, i}^{-}(2 l k+j)=\phi \\
X_{0, i}^{+}(2 l k+j)=X_{0, i}(2 l k+j)
\end{array}\right.
$$

when $l>d$ and $(-1)^{m+1} \tan \frac{(2 j+1) \pi}{4 k}>0$. Meanwhile,

$$
\left\{\begin{array}{l}
X_{\infty, i}^{-}(2 l k+j)=X_{\infty, i}(2 l k+j) \\
X_{0, i}^{+}(2 l k+j)=\phi \\
X_{\infty, i}^{+}(2 l k+j)=\phi \\
X_{0, i}^{-}(2 l k+j)=X_{0, i}(2 l k+j)
\end{array}\right.
$$

when $l>d$ and $(-1)^{m+1} \tan \frac{(2 j+1) \pi}{4 k}<0$.

So,

$$
\begin{aligned}
& X_{\infty}^{+}(2 l k+j) \bigcap X_{0}^{-}(2 l k+j)=C_{X(2 l k+j)}\left(X_{\infty}^{+}(2 l k+j) \bigcup X_{0}^{-}(2 l k+j)\right)=\phi, \\
& X_{\infty}^{-}(2 l k+j) \bigcap X_{0}^{+}(2 l k+j)=C_{X(2 l k+j)}\left(X_{\infty}^{-}(2 l k+j) \bigcup X_{0}^{+}(2 l k+j)\right)=\phi,
\end{aligned}
$$

when $l>d$. Thus, we have

$$
\begin{aligned}
& X_{\infty}^{+} \bigcap X_{0}^{-}=\bigoplus_{j=0}^{2 k-1} \stackrel{d}{\oplus}\left(X_{\infty}^{+}(2 l k+j) \bigcap X_{0}^{-}(2 l k+j)\right), \\
& X_{\infty}^{-} \bigcap X_{0}^{+}=\underset{j=0}{\stackrel{2 k-1}{d} \underset{l=0}{\oplus}}\left(X_{\infty}^{-}(2 l k+j) \bigcap X_{0}^{+}(2 l k+j)\right), \\
& C_{X}\left(X_{\infty}^{+} \bigcup X_{0}^{-}\right)=\bigoplus_{j=0}^{2 k-1} \bigoplus_{l=0}^{d}\left(C_{X(2 l k+j)}\left(X_{\infty}^{+}(2 l k+j) \bigcup X_{0}^{-}(2 l k+j)\right)\right), \\
& C_{X}\left(X_{\infty}^{-} \bigcup X_{0}^{+}\right)=\bigoplus_{j=0}^{2 k-1} \underset{l=0}{d}\left(C_{X(2 l k+j)}\left(X_{\infty}^{-}(2 l k+j) \bigcup X_{0}^{+}(2 l k+j)\right)\right) .
\end{aligned}
$$

Then, we can get

$$
\left\{\begin{array}{l}
\operatorname{dim}\left(X_{\infty}^{+} \cap X_{0}^{-}\right)<\infty, \\
\operatorname{cod}\left(X_{\infty}^{-} \cup X_{0}^{+}\right)=\operatorname{dim} C_{X}\left(X_{\infty}^{-} \cup X_{0}^{+}\right)<\infty, \\
\operatorname{dim}\left(X_{\infty}^{-} \cap X_{0}^{+}\right)<\infty, \\
\operatorname{cod}\left(X_{\infty}^{+} \cup X_{0}^{-}\right)=\operatorname{dim} C_{X}\left(X_{\infty}^{+} \cup X_{0}^{-}\right)<\infty
\end{array}\right.
$$


Denote

$$
\begin{aligned}
& K=\operatorname{dim}\left(X_{\infty}^{+} \bigcap X_{0}^{-}\right), \bar{K}_{c}=\operatorname{cod}\left(X_{\infty}^{+} \bigcup X_{0}^{-}\right), \\
& K_{-}=\operatorname{dim}\left(X_{\infty}^{-} \bigcap X_{0}^{+}\right), \bar{K}_{-c}=\operatorname{cod}\left(X_{\infty}^{-} \bigcup X_{0}^{+}\right) .
\end{aligned}
$$

Now we give the main results of this paper.

Theorem 1. Suppose that $\left(S_{1}\right)$ and $\left(S_{2}\right)$ hold. Then system (2) possesses at least

$$
\tilde{n}=\frac{1}{2} \max \left\{0, K-\bar{K}_{c}, K_{-}-\bar{K}_{-c}\right\}
$$

$4 k$-periodic orbits satisfying $x(t-2 k)=-x(t)$.

Proof of Theorem 1. Suppose without loss of generality that

$$
\tilde{n}=\frac{1}{2}\left[K-\bar{K}_{c}\right]>0
$$

Firstly, it can be seen from (16) that $K, \bar{K}_{c}, K_{-}$and $\bar{K}_{-c}$ are finite numbers, which implies that condition (a) in Lemma 1 holds. For each $i \in N$, we have that $x \in X(i)$ yields $\left(L+P^{-(2 m+1)} A_{\infty}\right) x \in$ $X(i)$ which implies that the condition (b) in Lemma 1 holds.

Moreover, Lemma 3 gives the (P.S)-condition.

In the following part, we just need to show that conditions (c) and (d) in Lemma 1 hold.

Let $\Pi_{i}: \mathbb{R}^{N} \rightarrow W_{i}, i=1,2, \cdots, N$ be the orthogonal projection. $\forall x \in X^{+}=X_{\infty}^{+}$, let $x_{i}=\Pi_{i} x \in$ $X^{+}$, then $x=\sum_{i=1}^{N} x_{i}, x_{i} \in X_{\infty, i}^{+}$.

The second condition in (4) implies that $\left|F(x)-\frac{1}{2}\left(A_{\infty} x, x\right)\right|<\frac{1}{4} \sigma|x|^{2}+M_{1}, x \in R$ for some $M_{1}>0$. Meanwhile, taking Lemma 2 into account, we can get

$$
\begin{aligned}
\Phi(x) & =\frac{1}{2}\langle L x, x\rangle+\int_{0}^{4 k} F(x(t)) d t \\
& =\frac{1}{2}\left\langle\left(L+P^{-(2 m+1)} A_{\infty}\right) x, x\right\rangle+\int_{0}^{4 k}\left[F(x(t))-\frac{1}{2}\left(A_{\infty} x, x\right)\right] d t \\
& \geq \frac{1}{2} \sigma\|x\|^{2}-\frac{1}{4} \sigma\|x\|^{2}-4 k M_{1} \\
& \geq \frac{1}{4} \sigma\|x\|^{2}-4 k M_{1}
\end{aligned}
$$

if $x \in X^{+}$. Clearly, there is $c_{0} \in R$ such that

$$
\inf _{x \in X^{+}} \Phi(x) \geq c_{0}
$$

On the other hand,

$$
\lim _{|x| \rightarrow 0} \frac{\left|F(x)-\frac{1}{2}\left(A_{0} x, x\right)\right|}{|x|^{2}}=\frac{1}{2} \lim _{|x| \rightarrow 0} \frac{\left|\nabla F(x)-A_{0} x\right|}{|x|}=0,
$$

so there exists $\delta>0$, such that

$$
\left|F(x)-\frac{1}{2}\left(A_{0} x, x\right)\right|<\frac{\sigma}{4}|x|^{2}, 0<|x| \leq \delta .
$$


Thus, $\exists r>0$, when $x \in X,\|x\|=r$, we have

$$
\left|F(x)-\frac{1}{2}\left(A_{0} x(t), x(t)\right)\right|<\frac{\sigma}{4}|| x \|^{2} .
$$

So

$$
\begin{aligned}
\Phi(x) & =\frac{1}{2}\langle L x, x\rangle+\int_{0}^{4 k} F(x(t)) d t \\
& =\frac{1}{2}\left\langle\left(L+P^{-(2 m+1)} A_{0}\right) x, x\right\rangle+\int_{0}^{4 k}\left[F(x(t))-\frac{1}{2}\left(A_{0} x, x\right)\right] d t \\
& \leq-\frac{1}{2} \sigma\|x\|^{2}+\frac{1}{4} \sigma\|x\|^{2} \\
& \leq-\frac{1}{4} \sigma\|x\|^{2} .
\end{aligned}
$$

That is, there are $r>0$ and $c_{\infty}<0$ such that

$$
\Phi(x) \leq c_{\infty}<0=\Phi(0), \forall x \in X^{-} \bigcap S_{r}=\{x \in X:\|x\|=r\} .
$$

Then, according to Lemma $1, \Phi(x)$ has at least $\tilde{n}$ different $4 k$-periodic orbits satisfying $x(t-2 k)=-x(t)$.

Furthermore, let

$$
\begin{aligned}
& n_{\infty, i}^{+}(2 l k+j)= \begin{cases}1, & (-1)^{m+1}\left(\frac{(4 l k+2 j+1) \pi}{2 k}\right)^{2 m+1} \tan \frac{(2 j+1) \pi}{4 k}>-\beta_{i}, \\
0, & (-1)^{m+1}\left(\frac{(4 l k+2 j+1) \pi}{2 k}\right)^{2 m+1} \tan \frac{(2 j+1) \pi}{4 k} \leq-\beta_{i},\end{cases} \\
& n_{\infty, i}^{-}(2 l k+j)= \begin{cases}1, & (-1)^{m+1}\left(\frac{(4 l k+2 j+1) \pi}{2 k}\right)^{2 m+1} \tan \frac{(2 j+1) \pi}{4 k}<-\beta_{i}, \\
0, & (-1)^{m+1}\left(\frac{(4 l k+2 j+1) \pi}{2 k}\right)^{2 m+1} \tan \frac{(2 j+1) \pi}{4 k} \geq-\beta_{i},\end{cases} \\
& n_{0, i}^{+}(2 l k+j)= \begin{cases}1, & (-1)^{m+1}\left(\frac{4 l k+2 j+1) \pi}{2 k}\right)^{2 m+1} \tan \frac{(2 j+1) \pi}{4 k}>-\alpha_{i}, \\
0, & (-1)^{m+1}\left(\frac{(4 l k+2 j+1) \pi}{2 k}\right)^{2 m+1} \tan \frac{(2 j+1) \pi}{4 k} \leq-\alpha_{i},\end{cases} \\
& n_{0, i}^{-}(2 l k+j)= \begin{cases}1, & (-1)^{m+1}\left(\frac{(4 l k+2 j+1) \pi}{2 k}\right)^{2 m+1} \tan \frac{(2 j+1) \pi}{4 k}<-\alpha_{i}, \\
0, & (-1)^{m+1}\left(\frac{(4 l k+2 j+1) \pi}{2 k}\right)^{2 m+1} \tan \frac{(2 j+1) \pi}{4 k} \geq-\alpha_{i},\end{cases}
\end{aligned}
$$

According to the definition of (13), we have

$$
\begin{aligned}
& n_{\infty, i}^{+}(2 l k+j)= \begin{cases}1, & X_{\infty, i}^{+}(2 l k+j)=X_{\infty, i}(2 l k+j), \\
0, & X_{\infty, i}^{+}(2 l k+j)=\phi,\end{cases} \\
& n_{\infty, i}^{-}(2 l k+j)=\left\{\begin{array}{cc}
1, & X_{\infty, i}^{-}(2 l k+j)=X_{\infty, i}(2 l k+j), \\
0, & X_{\infty, i}^{-}(2 l k+j)=\phi,
\end{array}\right. \\
& n_{0, i}^{+}(2 l k+j)=\left\{\begin{array}{cc}
1, & X_{0, i}^{+}(2 l k+j)=X_{0, i}(2 l k+j), \\
0, & X_{0, i}^{+}(2 l k+j)=\phi,
\end{array}\right. \\
& n_{0, i}^{-}(2 l k+j)=\left\{\begin{array}{cc}
1, & X_{0, i}^{-}(2 l k+j)=X_{0, i}(2 l k+j), \\
0, & X_{0, i}^{-}(2 l k+j)=\phi,
\end{array}\right.
\end{aligned}
$$

Let

$$
n_{0}^{ \pm}(2 l k+j)=\sum_{i=1}^{N} n_{0, i}^{ \pm}(2 l k+j), \quad n_{\infty}^{ \pm}(2 l k+j)=\sum_{i=1}^{N} n_{\infty, i}^{ \pm}(2 l k+j)
$$


Then, $\forall j \in\{0,1,2, \cdots, 2 k-1\}$ and $\forall i \in\{1,2, \cdots, N\}$,

$$
\left\{\begin{array}{ll}
n_{\infty, i}^{+}(2 l k+j)=1, & n_{0, i}^{-}(2 l k+j)=0, \\
n_{\infty, i}^{-}(2 l k+j)=0, & n_{0, i}^{+}(2 l k+j)=1,
\end{array} \quad(-1)^{m+1} \tan \frac{(2 j+1) \pi}{4 k}>0\right.
$$

when $l>d>0$. Therefore,

$$
\left\{\begin{array}{l}
n_{\infty}^{+}(2 l k+j)=N, \quad n_{0}^{-}(2 l k+j)=0, \\
n_{\infty}^{-}(2 l k+j)=0, \quad n_{0}^{+}(2 l k+j)=N,
\end{array} \quad(-1)^{m+1} \tan \frac{(2 j+1) \pi}{4 k}>0\right.
$$

when $l>d$.

On the other hand, $X_{\infty}^{+} \cap X_{0}^{-}=\underset{j=0}{2 k-1} \underset{l=0}{\oplus}\left(X_{\infty}^{+}(2 l k+j) \cap X_{0}^{-}(2 l k+j)\right)$, so

$$
\begin{aligned}
& \operatorname{dim}\left(X_{\infty}^{+} \bigcap X_{0}^{-}\right)-\operatorname{cod}_{X}\left(X_{\infty}^{+} \bigcup X_{0}^{-}\right) \\
= & \sum_{j=0}^{2 k-1} \sum_{l=0}^{d}\left[\operatorname{dim} X_{\infty}^{+}(2 l k+j)+\operatorname{dim} X_{0}^{-}(2 l k+j)-2 N\right] \\
= & 2 \sum_{j=0}^{2 k-1} \sum_{l=0}^{d}\left[n_{\infty}^{+}(2 l k+j)+n_{0}^{-}(2 l k+j)-N\right] .
\end{aligned}
$$

Similarly, $X_{\infty}^{-} \cap X_{0}^{+}=\underset{j=0}{2 k-1} \underset{l=0}{\oplus}\left(X_{\infty}^{-}(2 l k+j) \cap X_{0}^{+}(2 l k+j)\right)$, so

$$
\begin{aligned}
& \operatorname{dim}\left(X_{\infty}^{-} \bigcap X_{0}^{+}\right)-\operatorname{cod}_{X}\left(X_{\infty}^{-} \bigcup X_{0}^{+}\right) \\
= & 2 \sum_{j=0}^{2 k-1} \sum_{l=0}^{d}\left[n_{\infty}^{-}(2 l k+j)+n_{0}^{+}(2 l k+j)-N\right] .
\end{aligned}
$$

Hence,

$$
\begin{aligned}
K-\bar{K}_{c} & =\operatorname{dim}\left(X_{\infty}^{+} \bigcap X_{0}^{-}\right)-\operatorname{cod}_{X}\left(X_{\infty}^{+} \bigcup X_{0}^{-}\right) \\
& =\sum_{j=0}^{2 k-1} \sum_{l=0}^{d}\left[2 n_{\infty}^{+}(2 l k+j)+2 n_{0}^{-}(2 l k+j)-2 N\right], \\
K_{-}-\bar{K}_{-c} & =\sum_{j=0}^{2 k-1} \sum_{l=0}^{d}\left[2 n_{\infty}^{-}(2 l k+j)+2 n_{0}^{+}(2 l k+j)-2 N\right] .
\end{aligned}
$$

However, the existence of integer $d$ is proved only in theory in the above formulas, and the calculation method is not given, so it is difficult to calculate the specific values of $K-\bar{K}_{c}$ and $K_{-}-\bar{K}_{-c}$, causing inconvenience in practical application. So, define

$$
d_{j}=\left\{\begin{array}{l}
\min \left\{l \geq 0: \quad(-1)^{m+1}\left(\frac{(4 l k+2 j+1) \pi}{2 k}\right)^{2 m+1} \tan \frac{(2 j+1) \pi}{4 k}>-\gamma_{m}\right\}, \quad(-1)^{m+1} \tan \frac{(2 j+1) \pi}{4 k}>0 \\
\min \left\{l \geq 0: \quad(-1)^{m+1}\left(\frac{(4 l k+2 j+1) \pi}{2 k}\right)^{2 m+1} \tan \frac{(2 j+1) \pi}{4 k}<-\gamma_{M}\right\},(-1)^{m+1} \tan \frac{(2 j+1) \pi}{4 k}<0
\end{array}\right.
$$

in which $\gamma_{m}=\min \left\{\min _{1 \leq i \leq N} \alpha_{i}, \min _{1 \leq i \leq N} \beta_{i}\right\}, \gamma_{M}=\max \left\{\max _{1 \leq i \leq N} \alpha_{i}, \max _{1 \leq i \leq N} \beta_{i}\right\}$. 
Obviously, $d_{j} \leq d$. Then when $l>d_{j}$, we can get

$$
\begin{aligned}
& \left\{\begin{array}{l}
n_{\infty}^{+}(2 l k+j)=N, \quad n_{0}^{-}(2 l k+j)=0, \\
n_{\infty}^{-}(2 l k+j)=0, \quad n_{0}^{+}(2 l k+j)=N,
\end{array} \quad(-1)^{m+1} \tan \frac{(2 j+1) \pi}{4 k}>0,\right. \\
& \left\{\begin{array}{l}
n_{\infty}^{+}(2 l k+j)=0, \quad n_{0}^{-}(2 l k+j)=N, \\
n_{\infty}^{-}(2 l k+j)=N, \quad n_{0}^{+}(2 l k+j)=0,
\end{array} \quad(-1)^{m+1} \tan \frac{(2 j+1) \pi}{4 k}<0 .\right.
\end{aligned}
$$

Hence,

$$
\begin{gathered}
K-\bar{K}_{c}=\operatorname{dim}\left(X_{\infty}^{+} \bigcap X_{0}^{-}\right)-\operatorname{cod}\left(X_{\infty}^{+} \bigcup X_{0}^{-}\right) \\
=2 \sum_{j=0}^{2 k-1} \sum_{l=0}^{d_{j}}\left[n_{\infty}^{+}(2 l k+j)+n_{0}^{-}(2 l k+j)-N\right], \\
K_{-}-\bar{K}_{-c}=2 \sum_{j=0}^{2 k-1} \sum_{l=0}^{d_{j}}\left[n_{\infty}^{-}(2 l k+j)+n_{0}^{+}(2 l k+j)-N\right] .
\end{gathered}
$$

This leads to the following corollary,

Corollary 1. Suppose that $\left(S_{1}\right)$ and $\left(S_{2}\right)$ hold. Then system (2) possesses at least

$$
\tilde{n}=\max \left\{0, \sum_{j=0}^{2 k-1} \sum_{l=0}^{d_{j}}\left[n_{\infty}^{+}(2 l k+j)+n_{0}^{-}(2 l k+j)-N\right], \sum_{j=0}^{2 k-1} \sum_{l=0}^{d_{j}}\left[n_{\infty}^{-}(2 l k+j)+n_{0}^{+}(2 l k+j)-N\right]\right\}
$$

geometrically different $4 k$-periodic orbits satisfying $x(t-2 k)=-x(t)$.

Especially, when the system (2) can be decomposed into n systems, let

$$
z=\left(y_{1}, y_{2}, \cdots, y_{n}\right), r_{0}=0,
$$

and

$$
y_{i}^{(2 m+1)}(t)=-\sum_{p=1}^{2 k-1} \nabla \tilde{H}\left(y_{i}(t-p)\right), \quad y_{i} \in \mathbb{R}_{i}^{r_{i}-r_{i-1}}, i=1,2, \cdots, n
$$

in which $y_{i}=\left(x_{r_{i-1}+1}, x_{r_{i-1}+2}, \cdots, x_{r_{i}}\right), \nabla \tilde{H}\left(y_{i}\right)=\nabla_{y_{i}} H\left(0, \cdots, 0, y_{i}, 0, \cdots, 0\right)$.

It is easy to see that (18) is a $\left(r_{i}-r_{i-1}\right)$-dimensional system. From (4), we can get

$$
\begin{aligned}
& \left|\nabla \tilde{H}\left(y_{i}\right)-B_{0, i} y_{i}\right|=\circ\left(\left|y_{i}\right|\right), \quad\left|y_{i}\right| \rightarrow 0, \\
& \left|\nabla \tilde{H}\left(y_{i}\right)-B_{\infty, i} y_{i}\right|=\circ\left(\left|y_{i}\right|\right), \quad\left|y_{i}\right| \rightarrow \infty .
\end{aligned}
$$

Assume that

$$
\sigma\left(B_{0, i}\right)=\left(\alpha_{r_{i-1}+1}, \alpha_{r_{i-1}+2}, \cdots, \alpha_{r_{i}}\right), \quad \text { and } \quad \sigma\left(B_{\infty, i}\right)=\left(\beta_{r_{i-1}+1}, \beta_{r_{i-1}+2}, \cdots, \beta_{r_{i}}\right)
$$

are respectively the eigenvalues of $B_{0, i}$ and $B_{\infty, i}$. Their corresponding unit eigenvectors in space are respectively $\left(u_{r_{i-1}+1}, u_{r_{i-1}+2}, \cdots, u_{r_{i}}\right)$ and $\left(v_{r_{i-1}+1}, v_{r_{i-1}+2}, \cdots, v_{r_{i}}\right)$. 
For (18), let

$$
\begin{aligned}
X^{i}=c l\{x(t)= & \sum_{j=0}^{\infty} a_{j} \cos \frac{(2 j+1) \pi t}{2 k}+b_{j} \sin \frac{(2 j+1) \pi t}{2 k}: a_{j}, b_{j} \in \mathbb{R}^{r_{i}-r_{i-1}}, \\
& \left.\sum_{j=0}^{\infty}(2 j+1)^{2 m+1}\left(\left|a_{j}\right|^{2}+\left|b_{j}\right|^{2}\right)<\infty\right\}
\end{aligned}
$$

and

$$
X^{i}(j)=\left\{a_{j} \cos \frac{(2 j+1) \pi t}{2 k}+b_{j} \sin \frac{(2 j+1) \pi t}{2 k}: a_{j}, b_{j} \in \mathbb{R}^{r_{i}-r_{i-1}}\right\},
$$

then

$$
X^{i}=\bigoplus_{j=0}^{\infty} X^{i}(j)=\bigoplus_{l=0}^{\infty} \underset{j=0}{2 k-1} X^{i}(2 l k+j)
$$

Further subdivision, let

$$
\begin{array}{r}
X_{0, s}^{i}(j)=\left\{a_{j} \cos \frac{(2 j+1) \pi t}{2 k}+b_{j} \sin \frac{(2 j+1) \pi t}{2 k}: a_{j}, b_{j} \in U_{s}\right\}, \\
X_{\infty, s}^{i}(j)=\left\{a_{j} \cos \frac{(2 j+1) \pi t}{2 k}+b_{j} \sin \frac{(2 j+1) \pi t}{2 k}: a_{j}, b_{j} \in V_{s}\right\}, \\
X^{i}=\bigoplus_{j=0}^{\infty} \underset{s=r_{i-1}+1}{\overbrace{i}} X_{0, s}^{i}(j)=\bigoplus_{j=0}^{\infty} \underset{s=r_{i-1}+1}{r_{i}} X_{\infty, s}^{i}(j) .
\end{array}
$$

$\forall s \in\left\{r_{i-1}+1, r_{i-1}+2, \cdots, r_{i}\right\}$, denote

$$
\begin{aligned}
& X_{0, s}^{i,+}=\oplus\left\{X_{0, s}^{i}(2 l k+j):(-1)^{m+1}\left(\frac{\pi}{2 k}\right)^{2 m+1}(4 l k+2 j+1)^{2 m+1} \tan \frac{(2 j+1) \pi}{4 k}+\alpha_{s}>0\right\}, \\
& X_{0, s}^{i, 0}=\oplus\left\{X_{0, s}^{i}(2 l k+j):(-1)^{m+1}\left(\frac{(4 l k+2 j+1) \pi}{2 k}\right)^{2 m+1} \tan \frac{(2 j+1) \pi}{4 k}=-\alpha_{s}\right\}, \\
& X_{0, s}^{i,-}=\oplus\left\{X_{0, s}^{i}(2 l k+j):(-1)^{m+1}\left(\frac{\pi}{2 k}\right)^{2 m+1}(4 l k+2 j+1)^{2 m+1} \tan \frac{(2 j+1) \pi}{4 k}+\alpha_{s}<0\right\} \\
& X_{\infty, s}^{i,+}=\oplus\left\{X_{\infty, s}^{i}(2 k+j):(-1)^{m+1}\left(\frac{\pi}{2 k}\right)^{2 m+1}(4 k+2 j+1)^{2 m+1} \tan \frac{(2 j+1) \pi}{4 k}+\beta_{s}>0\right\}, \\
& X_{\infty, s}^{i, 0}=\oplus\left\{X_{\infty, s}^{i}(2 k+j):(-1)^{m+1}\left(\frac{(4 k+2 j+1) \pi}{2 k}\right)^{2 m+1} \tan \frac{(2 j+1) \pi}{4 k}=-\beta_{s}\right\}, \\
& X_{\infty, s}^{i,-}=\oplus\left\{X_{\infty, s}^{i}(2 k+j):(-1)^{m+1}\left(\frac{\pi}{2 k}\right)^{2 m+1}(4 k+2 j+1)^{2 m+1} \tan \frac{(2 j+1) \pi}{4 k}+\beta_{s}<0\right\},
\end{aligned}
$$

and

$$
\begin{aligned}
X_{0}^{i,+} & =\underset{s=r_{i-1}+1}{r_{i}} X_{0, s}^{i,+}, X_{0}^{i, 0}=\underset{s=r_{i-1}+1}{r_{i}} X_{0, s}^{i, 0}, X_{0}^{i,-}=\underset{s=r_{i-1}+1}{r_{i}} X_{0, s}^{i,-}, \\
X_{\infty}^{i,+} & =\underset{s=r_{i-1}+1}{r_{i}} X_{\infty, s}^{i,+}, X_{\infty}^{i, 0}=\underset{s=r_{i-1}+1}{r_{i}} X_{\infty, s}^{i, 0}, X_{\infty}^{i,-}=\underset{s=r_{i-1}+1}{r_{i}} X_{\infty, s}^{i,-}, \\
K^{i} & =\operatorname{dim}\left(X_{\infty}^{i,+} \bigcap X_{0}^{i,-}\right), \bar{K}_{c}^{i}=\operatorname{cod}\left(X_{\infty}^{i,+} \bigcup X_{0}^{i,-}\right), \\
K^{i}- & =\operatorname{dim}\left(X_{\infty}^{i,-} \bigcap X_{0}^{i,+}\right), \bar{K}_{-c}=\operatorname{cod}\left(X_{\infty}^{i,-} \bigcup X_{0}^{i,+}\right) .
\end{aligned}
$$

Then we can get 
Theorem 2. Suppose that $\left(S_{1}\right)$ and $\left(S_{2}\right)$ hold and the differential system (2) can be decomposed into the independent systems shown in (18), then the system (2) possesses at least

$$
\tilde{n}=\frac{1}{2} \sum_{i=1}^{n} \max \left\{0, K^{i}-\bar{K}_{c}^{i}, K_{-}^{i}-\bar{K}_{-c}^{i}\right\}
$$

geometrically different $4 k$-periodic orbits satisfying $x(t-2 k)=-x(t)$.

Proof of Theorem 2. If the system (2) can be decomposed into $\mathrm{n}$ subsystems as shown in (18), according to Theorem 1 , each subsystem has at least

$$
\tilde{n}^{i}=\frac{1}{2} \max \left\{0, K^{i}-\bar{K}_{c}^{i}, K^{i}-\bar{K}_{-c}^{i}\right\}, i \in\{1,2, \cdots, n\}
$$

$4 k$-periodic orbits satisfying $x(t-2 k)=-x(t)$. However, the periodic orbits of each different system represented by (18) are different and all of them are periodic orbits of (2). Therefore, the conclusion of Theorem 2 is given.

Furthermore, when $r_{i-1}+1 \leq s \leq r_{i}\left(r_{0}=0, r_{n}=N\right)$, let

$$
\begin{gathered}
n_{\infty, s}^{i,+}(2 l k+j)= \begin{cases}1, & (-1)^{m+1}\left(\frac{(4 l k+2 j+1) \pi}{2 k}\right)^{2 m+1} \tan \frac{(2 j+1) \pi}{4 k}>-\beta_{s}, \\
0, & (-1)^{m+1}\left(\frac{(4 l k+2 j+1) \pi}{2 k}\right)^{2 m+1} \tan \frac{(2 j+1) \pi}{4 k} \leq-\beta_{s},\end{cases} \\
n_{\infty, s}^{i,-}(2 l k+j)= \begin{cases}1, & (-1)^{m+1}\left(\frac{(4 l k+2 j+1) \pi}{2 k}\right)^{2 m+1} \tan \frac{(2 j+1) \pi}{4 k}<-\beta_{s}, \\
0, & (-1)^{m+1}\left(\frac{4 l k+2 j+1) \pi}{2 k}\right)^{2 m+1} \tan \frac{(2 j+1) \pi}{4 k} \geq-\beta_{s} ;\end{cases} \\
n_{0, s}^{i,+}(2 l k+j)= \begin{cases}1, & (-1)^{m+1}\left(\frac{(4 l k+2 j+1) \pi}{2 k}\right)^{2 m+1} \tan \frac{(2 j+1) \pi}{4 k}>-\alpha_{s}, \\
0, & (-1)^{m+1}\left(\frac{(4 l k+2 j+1) \pi}{2 k}\right)^{2 m+1} \tan \frac{(2 j+1) \pi}{4 k} \leq-\alpha_{s},\end{cases} \\
n_{0, s}^{i,-}(2 l k+j)= \begin{cases}1, & (-1)^{m+1}\left(\frac{(4 l k+2 j+1) \pi}{2 k}\right)^{2 m+1} \tan \frac{(2 j+1) \pi}{4 k}<-\alpha_{s}, \\
0, & (-1)^{m+1}\left(\frac{(4 l k+2 j+1) \pi}{2 k}\right)^{2 m+1} \tan \frac{(2 j+1) \pi}{4 k} \geq-\alpha_{s},\end{cases} \\
n_{\infty}^{i,+}(2 l k+j)=\sum_{s=r_{i-1}+1}^{r_{i}} n_{\infty, s}^{i,+}(2 l k+j), n_{\infty}^{i,-}(2 l k+j)=\sum_{s=r_{i-1}+1}^{r_{i}} n_{\infty, s}^{i,-}(2 l k+j),
\end{gathered}
$$

Then for $j \in\{0,1,2, \cdots, 2 k-1\}, i \in\{1,2, \cdots, n\}, s \in\left\{r_{i-1}+1, r_{i-1}+2, \cdots, r_{i}\right\}$, define

$$
d_{j}^{i}=\left\{\begin{array}{l}
\min \left\{l \geq 0:(-1)^{m+1}\left(\frac{(4 l k+2 j+1) \pi}{2 k}\right)^{2 m+1} \tan \frac{(2 j+1) \pi}{4 k}>-\gamma_{m}^{i}\right\}, \quad(-1)^{m+1} \tan \frac{(2 j+1) \pi}{4 k}>0 \\
\min \left\{l \geq 0:(-1)^{m+1}\left(\frac{(4 l k+2 j+1) \pi}{2 k}\right)^{2 m+1} \tan \frac{(2 j+1) \pi}{4 k}<-\gamma_{M}^{i}\right\}, \quad(-1)^{m+1} \tan \frac{(2 j+1) \pi}{4 k}<0
\end{array}\right.
$$

in which $\gamma_{m}^{i}=\min \left\{\min _{r_{i-1}+1 \leq i \leq r_{i}} \alpha_{i}, \min _{r_{i-1}+1 \leq i \leq r_{i}} \beta_{i}\right\}, \gamma_{M}^{i}=\max \left\{\max _{r_{i-1}+1 \leq i \leq r_{i}} \alpha_{i}, \max _{r_{i-1}+1 \leq i \leq r_{i}} \beta_{i}\right\}$. Obviously, $d_{j}^{i} \leq d$. Then when $l>d_{j}^{i}$, we have 


$$
\begin{gathered}
\left\{\begin{array}{ll}
n_{\infty, s}^{i,+}(2 l k+j)=1, & n_{0, s}^{i,-}(2 l k+j)=0, \\
n_{\infty, s}^{i,-}(2 l k+j)=0, & n_{0, s}^{i,+}(2 l k+j)=1,
\end{array} \quad(-1)^{m+1} \tan \frac{(2 j+1) \pi}{4 k}>0,\right. \\
\left\{\begin{array}{ll}
n_{\infty, s}^{i,+}(2 l k+j)=0, & n_{0, s}^{i,-}(2 l k+j)=1, \\
n_{\infty, s}^{i,-}(2 l k+j)=1, & n_{0, s}^{i,+}(2 l k+j)=0,
\end{array} \quad(-1)^{m+1} \tan \frac{(2 j+1) \pi}{4 k}<0,\right. \\
n_{\infty}^{i,+}(2 l k+j)+n_{0}^{i,-}(2 l k+j)=r_{i}-r_{i-1}, \\
n_{\infty}^{i,-}(2 l k+j)+n_{0}^{i,+}(2 l k+j)=r_{i}-r_{i-1} .
\end{gathered}
$$

Hence,

$$
\begin{aligned}
& K^{i}-\bar{K}_{c}^{i}=\operatorname{dim}\left(X_{\infty}^{+} \bigcap X_{0}^{-}\right)-\operatorname{cod}\left(X_{\infty}^{+} \bigcup X_{0}^{-}\right) \\
& =\sum_{j=0}^{2 k-1} \sum_{l=0}^{d_{j}^{i}}\left[2 n_{\infty}^{i,+}(2 l k+j)+2 n_{0}^{i,-}(2 l k+j)-2\left(r_{i}-r_{i-1}\right)\right] \\
& K_{-}^{i}-\bar{K}_{-c}^{i}=\sum_{j=0}^{2 k-1} \sum_{l=0}^{d_{j}^{i}}\left[2 n_{\infty}^{i,-}(2 l k+j)+2 n_{0}^{i,+}(2 l k+j)-2\left(r_{i}-r_{i-1}\right)\right]
\end{aligned}
$$

Combining theorem 2 with corollary 1 , it is easy to get

Corollary 2. Suppose that $\left(S_{1}\right)$ and $\left(S_{2}\right)$ hold and the differential system (2) can be decomposed into the independent systems shown in (18), then the system (2) possesses at least

$$
\begin{aligned}
\tilde{n}=\sum_{i=1}^{n} \max \{0 & 0, \sum_{j=0}^{2 k-1} \sum_{l=0}^{d_{j}^{i}}\left[n_{\infty}^{i,+}(2 l k+j)+n_{0}^{i,-}(2 l k+j)-\left(r_{i}-r_{i-1}\right)\right], \\
& \left.\sum_{j=0}^{2 k-1} \sum_{l=0}^{d_{j}^{i}}\left[n_{\infty}^{i,-}(2 l k+j)+n_{0}^{i,+}(2 l k+j)-\left(r_{i}-r_{i-1}\right)\right]\right\}
\end{aligned}
$$

geometrically different $4 k$-periodic orbits satisfying $x(t-2 k)=-x(t)$.

\section{Example}

Example 1. We study the multiplicity of 8-periodic orbits of the system

$$
x^{(3)}(t)=-\sum_{q=1}^{3} \nabla F(x(t-q)), \quad x \in \mathbb{R}^{4}
$$

in which

$$
\begin{gathered}
F(x)=\frac{\left(A_{0} x, x\right)+\left(A_{\infty} x, x\right) \sum_{i=1}^{4} x_{i}^{2}}{2\left(1+\sum_{i=1}^{4} x_{i}^{2}\right)}, \quad x=\left(x_{1}, x_{2}, \cdots, x_{4}\right) \\
A_{0}=\pi^{3}\left(\begin{array}{cccc}
-12 & 0 & 0 & 0 \\
0 & -10 & 0 & 0 \\
0 & 0 & 33 & 2 \\
0 & 0 & 2 & 36
\end{array}\right), A_{\infty}=\pi^{3}\left(\begin{array}{cccc}
12 & 0 & 0 & 0 \\
0 & 15 & 0 & 0 \\
0 & 0 & -6 & 2 \\
0 & 0 & 2 & -9
\end{array}\right) .
\end{gathered}
$$


In this case,

$$
\begin{gathered}
\alpha_{1}=-12 \pi^{3}, \alpha_{2}=-10 \pi^{3}, \alpha_{3}=32 \pi^{3}, \alpha_{4}=37 \pi^{3}, \\
u_{1}=(1,0,0,0), u_{2}=(0,1,0,0), u_{3}=\left(0,0, \frac{2}{\sqrt{5}}, \frac{-1}{\sqrt{5}}\right), u_{4}=\left(0,0, \frac{1}{\sqrt{5}}, \frac{2}{\sqrt{5}}\right), \\
\beta_{1}=12 \pi^{3}, \beta_{2}=15 \pi^{3}, \beta_{3}=-5 \pi^{3}, \beta_{4}=-10 \pi^{3}, \\
v_{1}=(1,0,0,0), v_{2}=(0,1,0,0), v_{3}=\left(0,0, \frac{2}{\sqrt{5}}, \frac{1}{\sqrt{5}}\right), v_{4}=\left(0,0, \frac{1}{\sqrt{5}}, \frac{-2}{\sqrt{5}}\right) .
\end{gathered}
$$

Then

$$
W_{1}=\operatorname{span}\left\{u_{1}\right\}=\operatorname{span}\left\{v_{1}\right\}, W_{2}=\operatorname{span}\left\{u_{2}\right\}=\operatorname{span}\left\{v_{2}\right\}, \quad W_{3}=\operatorname{span}\left\{u_{3}, u_{4}\right\}=\operatorname{span}\left\{v_{3}, v_{4}\right\},
$$

and $\operatorname{dim} W_{1}=\operatorname{dim} W_{2}=1, \operatorname{dim} W_{3}=2$.

Let

$$
\begin{aligned}
F_{1}\left(x_{1}\right) & =\pi^{3}\left[\frac{-6 x_{1}^{2}+6 x_{1}^{4}}{1+x_{1}^{2}}\right], \quad F_{2}\left(x_{2}\right)=\pi^{3}\left[\frac{-10 x_{2}^{2}+15 x_{2}^{4}}{2\left(1+x_{2}^{2}\right)}\right] \\
F_{3}\left(x_{3}, x_{4}\right) & =\pi^{3}\left[\frac{33 x_{3}^{2}+4 x_{3} x_{4}+36 x_{4}^{2}+\left(-6 x_{3}^{2}+4 x_{3} x_{4}-9 x_{4}^{2}\right)\left(x_{3}^{2}+x_{4}^{2}\right)}{2\left(1+x_{3}^{2}+x_{4}^{2}\right)}\right] .
\end{aligned}
$$

Then

$$
\begin{gathered}
\nabla F_{1}=\pi^{3}\left(\frac{-12 x_{1}+36 x_{1}^{3}}{1+x_{1}^{2}}-\frac{24 x_{1}^{5}}{\left(1+x_{1}^{2}\right)^{2}}\right), \nabla F_{2}=\pi^{3}\left(\frac{-10 x_{1}+40 x_{1}^{3}}{1+x_{2}^{2}}-\frac{25 x_{1}^{3}}{\left(1+x_{2}^{2}\right)^{2}}\right), \\
\nabla F_{3}=\pi^{3}\left(\frac{33 x_{3}+2 x_{4}+\left(-6 x_{3}+2 x_{4}\right)\left(x_{3}^{2}+x_{4}^{2}\right)}{1+x_{3}^{2}+x_{4}^{2}}+\frac{x_{3}\left(-6 x_{3}^{2}+4 x_{3} x_{4}-9 x_{4}^{2}\right)}{\left(1+x_{3}^{2}+x_{4}^{2}\right)^{2}},\right. \\
\left.\frac{2 x_{3}+36 x_{4}+\left(-6 x_{3}+2 x_{4}\right)\left(x_{3}^{2}+x_{4}^{2}\right)}{1+x_{3}^{2}+x_{4}^{2}}-\frac{x_{4}\left(-6 x_{3}^{2}+4 x_{3} x_{4}-9 x_{4}^{2}\right)}{\left(1+x_{3}^{2}+x_{4}^{2}\right)^{2}}\right) .
\end{gathered}
$$

System (19) can be decomposed into independent systems as follows

$$
\begin{aligned}
& x_{1}^{(3)}(t)=-\sum_{q=1}^{3} \nabla F_{1}\left(x_{1}(t-q)\right), \quad x_{1} \in \mathbb{R}, \\
& x_{2}^{(3)}(t)=-\sum_{q=1}^{3} \nabla F_{3}\left(x_{2}(t-q)\right), \quad x_{2} \in \mathbb{R}, \\
& \left(x_{3}^{(3)}(t), x_{4}^{(3)}(t)\right)=-\sum_{q=1}^{3} \nabla F_{2}\left(x_{3}(t-q), x_{4}(t-q)\right), \quad\left(x_{3}, x_{4}\right) \in \mathbb{R}^{2} .
\end{aligned}
$$

Hence, according to corollary 2, we can get

$$
\begin{aligned}
\tilde{n} & =\max \left\{0, \sum_{j=0}^{3} \sum_{l=0}^{d_{j}^{1}}\left[n_{\infty}^{1,+}(4 l+j)+n_{0}^{1,-}(4 l+j)-1\right], \sum_{j=0}^{3} \sum_{l=0}^{d_{j}^{1}}\left[n_{\infty}^{1,-}(4 l+j)+n_{0}^{1,+}(4 l+j)-1\right]\right\} \\
& +\max \left\{0, \sum_{j=0}^{3} \sum_{l=0}^{d_{j}^{2}}\left[n_{\infty}^{2,+}(4 l+j)+n_{0}^{2,-}(4 l+j)-1\right], \sum_{j=0}^{3} \sum_{l=0}^{d_{j}^{2}}\left[n_{\infty}^{2,-}(4 l+j)+n_{0}^{2,+}(4 l+j)-1\right]\right\} \\
& +\max \left\{0, \sum_{j=0}^{3} \sum_{l=0}^{d_{j}^{3}}\left[n_{\infty}^{3,+}(4 l+j)+n_{0}^{3,-}(4 l+j)-2\right], \sum_{j=0}^{3} \sum_{l=0}^{d_{j}^{3}}\left[n_{\infty}^{3,-}(4 l+j)+n_{0}^{3,+}(4 l+j)-2\right]\right\} .
\end{aligned}
$$


In this case, $m=1, k=2,(-1)^{m+1}=1, j \in\{0,1,2,3\}$, then by calculating, we have

$$
\begin{aligned}
& \tan \frac{\pi}{8}=0.4142, \tan \frac{3 \pi}{8}=2.4142, \quad \tan \frac{5 \pi}{8}=-2.4142, \tan \frac{7 \pi}{8}=-0.4142 . \\
& \gamma_{m}^{1}=-12 \pi^{3}, \gamma_{M}^{1}=12 \pi^{3}, \quad \gamma_{m}^{2}=-10 \pi^{3}, \gamma_{M}^{2}=15 \pi^{3}, \quad \gamma_{m}^{3}=-10 \pi^{3}, \gamma_{M}^{3}=37 \pi^{3} . \\
& d_{0}^{1}=\min \left\{l \geq 0: 0.4142\left(\frac{8 l+1}{4}\right)^{3}>12\right\}=2, \\
& d_{1}^{1}=\min \left\{l \geq 0: \quad 2.4142\left(\frac{8 l+3}{4}\right)^{3}>12\right\}=1, \\
& d_{2}^{1}=\min \left\{l \geq 0: \quad-2.4142\left(\frac{8 l+5}{4}\right)^{3}<-12\right\}=1, \\
& d_{3}^{1}=\min \left\{l \geq 0: \quad-0.4142\left(\frac{8 l+7}{4}\right)^{3}<-12\right\}=1, \\
& d_{0}^{2}=\min \left\{l \geq 0: \quad 0.4142\left(\frac{8 l+1}{4}\right)^{3}>10\right\}=2, \\
& d_{1}^{2}=\min \left\{l \geq 0: \quad 2.4142\left(\frac{8 l+3}{4}\right)^{3}>10\right\}=1, \\
& d_{2}^{2}=\min \left\{l \geq 0: \quad-2.4142\left(\frac{8 l+5}{4}\right)^{3}<-15\right\}=1, \\
& d_{3}^{2}=\min \left\{l \geq 0: \quad-0.4142\left(\frac{8 l+7}{4}\right)^{3}<-15\right\}=1, \\
& d_{0}^{3}=\min \left\{l \geq 0: \quad 0.4142\left(\frac{8 l+1}{4}\right)^{3}>10\right\}=2, \\
& d_{1}^{3}=\min \left\{l \geq 0: \quad 2.4142\left(\frac{8 l+3}{4}\right)^{3}>10\right\}=1, \\
& d_{2}^{3}=\min \left\{l \geq 0: \quad-2.4142\left(\frac{8 l+5}{4}\right)^{3}<-37\right\}=1, \\
& d_{3}^{3}=\min \left\{l \geq 0: \quad-0.4142\left(\frac{8 l+7}{4}\right)^{3}<-37\right\}=2 .
\end{aligned}
$$

and

$$
\begin{aligned}
& j=0, \\
& n_{\infty, 1}^{1,+}(0)=n_{\infty, 1}^{1,+}(4)=n_{\infty, 1}^{1,+}(8)=1, \quad n_{0,1}^{1,-}(0)=n_{0,1}^{1,-}(4)=1, n_{0,1}^{1,-}(8)=0, \\
& n_{\infty, 2}^{2,+}(0)=n_{\infty, 2}^{2,+}(4)=n_{\infty, 2}^{2,+}(8)=1, \quad n_{0,2}^{2,-}(0)=n_{0,2}^{2,-}(4)=1, n_{0,2}^{2,-}(8)=0 \\
& n_{\infty, 3}^{3,+}(0)=n_{\infty, 3}^{3,+}(4)=0, n_{\infty, 3}^{3,+}(8)=1, \quad n_{0,3}^{3,-}(0)=n_{0,3}^{3,-}(4)=n_{0,3}^{3,-}(8)=1 \\
& n_{\infty, 4}^{3,+}(0)=n_{\infty, 4}^{3,+}(4)=0, n_{\infty, 4}^{3,+}(8)=1, \quad n_{0,4}^{3,-}(0)=n_{0,4}^{3,-}(4)=n_{0,4}^{3,-}(8)=0 . \\
& j=1, \\
& n_{\infty, 1}^{1,+}(1)=1, \quad n_{\infty, 1}^{1,+}(5)=1, \quad n_{0,1}^{1,-}(1)=1, n_{0,1}^{1,-}(5)=0, \\
& n_{\infty, 2}^{2,+}(1)=1, \quad n_{\infty, 2}^{2,+}(5)=1, \quad n_{0,2}^{2,-}(1)=1, n_{0,2}^{2,-}(5)=0, \\
& n_{\infty, 3}^{3,+}(1)=0, n_{\infty, 3}^{3,+}(5)=1, \quad n_{0,3}^{3,-}(1)=0, \quad n_{0,3}^{3,-}(5)=0, \\
& n_{\infty, 4}^{3,+}(1)=0, n_{\infty, 4}^{3,+}(5)=1, \quad n_{0,4}^{3,-}(1)=0, \quad n_{0,4}^{3,-}(5)=0 . \\
& j=2, \\
& n_{\infty, 1}^{1,+}(2)=1, n_{\infty, 1}^{1,+}(6)=0, \quad n_{0,1}^{1,-}(2)=1, n_{0,1}^{1,-}(6)=1, \\
& n_{\infty, 2}^{2,+}(2)=1, n_{\infty, 2}^{2,+}(6)=0, \quad n_{0,2}^{2,-}(2)=1, n_{0,2}^{2,-}(6)=1, \\
& n_{\infty, 3}^{3,+}(2)=n_{\infty, 3}^{3,+}(6)=0, \quad n_{0,3}^{3,-}(2)=0, n_{0,3}^{3,-}(6)=1, \\
& n_{\infty, 4}^{3,+}(2)=n_{\infty, 4}^{3,+}(6)=0, \quad n_{0,4}^{3,-}(2)=0, n_{0,4}^{3,-}(6)=1 .
\end{aligned}
$$




$$
\begin{aligned}
& j=3, \\
& n_{\infty, 1}^{1,+}(3)=1, n_{\infty, 1}^{1,+}(7)=0, \quad n_{0,1}^{1,-}(3)=1, \quad n_{0,1}^{1,-}(7)=1, \\
& n_{\infty, 2}^{2,+}(3)=1, n_{\infty, 2}^{2,+}(7)=0, \quad n_{0,2}^{2,-}(3)=1, n_{0,2}^{2,-}(7)=1, \\
& n_{\infty, 3}^{3,+}(3)=n_{\infty, 3}^{3,+}(7)=n_{\infty, 3}^{3,+}(10)=0, \quad n_{0,3}^{3,-}(3)=n_{0,3}^{3,-}(7)=0, n_{0,3}^{3,-}(10)=1, \\
& n_{\infty, 4}^{3,+}(3)=n_{\infty, 4}^{3,+}(7)=n_{\infty, 4}^{3,+}(10)=0, \quad n_{0,4}^{3,-}(3)=n_{0,4}^{3,-}(7)=0, n_{0,4}^{3,-}(10)=1 .
\end{aligned}
$$

So, according to corollary 2, system (19) possesses at least $\tilde{n}=10+10+3=23$ geometrically different 8-periodic orbits satisfying $x(t-4)=-x(t)$.

Author Contributions: Both authors contributed equally and significantly in writing this paper. Both authors have read and agreed to the published version of the manuscript.

Funding: This research was funded by the National Science Foundations of China grant number 11601493.

Acknowledgments: The authors thank the referees for carefully reading the manuscript and for their valuable suggestions, which have significantly improved the paper.

Conflicts of Interest: The authors declare no conflict of interest.

\section{References}

1. Kolmanovskii, V.; Myshkis, A. Introduction to the Theory and Applications of Functional Differential Equations; Kluver: Dordrecht, The Netherlands, 1999.

2. Smith, H.L. An Introduction to Delay Differential Equations with Applications to the Life Sciences; Springer: New York, NY, USA, 2011.

3. Kaplan,J.; Yorke, J. Ordinary differential equations which yield periodic solution of delay equations. J. Math. Anal. Appl. 1974, 48, 317-324. [CrossRef]

4. Fei, G. Multiple periodic solutions of differential delay equations via Hamiltonian systems (I). Nonlinear Anal 2006, 65, 25-39. [CrossRef]

5. Fei, G. Multiple periodic solutions of differential delay equations via Hamiltonian systems (II). Nonlinear Anal 2006, 65, 40-58. [CrossRef]

6. Sun, Z.; Ge, W.; Li, L. Multiple periodic orbits of high-dimensional differential delay systems. Adv. Diff. Equ. 2019, 2019, 488. [CrossRef]

7. Ge, W. Periodic solutions of the differential delay equation $x^{\prime}(t)=-f(x(t-1))$. Acta Math. Sin. (New Ser.) 1996, 12, 113-121.

8. Ge, W. On the existence of periodic solutions of differential delay equations with multiple lags. Acta Appl. Math. Sin. 1994, 17, 173-181. (In Chinese)

9. Ge, W. Two existence theorems of periodic solutions for differential delay equations. Chin. Ann. Math. 1994, $15,217-224$.

10. Ge, W. Oscillatory periodic solutions of differential delay equations with multiple lags. Chin. Sci. Bull. 1997, 42, 444-447. [CrossRef]

11. Ge, W.; Zhang, L. Multiple periodic solutions of delay differential systems with $2 k-1$ lags via variational approach. Discret. Contin. Dyn. Syst. 2016, 36, 4925-4943. [CrossRef]

12. Guo, Z.; Yu, J. Multiple results on periodic solutions to higher dimensional differential equations with multiple delays. J. Dyn. Diff. Equ. 2011, 23, 1029-1052. [CrossRef]

13. Guo, Z.; Yu, J. Multiplicity results for periodic solutions to delay differential equations via critical point theory. J. Differ. Equ. 2005, 218, 15-35. [CrossRef]

14. Li, L; Xue, C.; Ge, W. Periodic orbits to Kaplan-Yorke like differential delay equations with two lags of ratio. Adv. Differ. Equ. 2016, 247.

15. Li, J.; He, X. Multiple periodic solutions of differential delay equations created by asymptotically linear Hamiltonian systems. Nonlinear Analysis. Theory Methods Appl. 1998, 31, 45-54. [CrossRef]

16. Li, L.; Sun, H.; Ge, W. Multiple Periodic Solutions of Differential Delay Equations with $2 k-1$ Lags. Acta Math. Appl. Sin. Engl. Ser. 2020, 36, 390-400. [CrossRef] 
17. Li, L.; Sun, H.; Ge, W. On the Number of Periodic Solutions to Kaplan Yorke like High Order Differential Delay Equations with 2 k Lags. Int. J. Bifurc. Chaos 2019, 29, 1950196. [CrossRef]

18. Nussbaum, R. Periodic solutions of special differential delay equations: An example in nonlinear functional analysis. Proc. R. Soc. Edingb. 1978, 81, 131-151. [CrossRef]

19. Benci, V. On critical point theory for indefinite functionals in the presence of symmetries. Trans. Am. Math. Soc. 1982, 274, 533-572. [CrossRef]

20. Fannio, L. Multiple periodic solutions of Hamiltonian systems with strong resonance at infinity. Discret. Cont. Dyn. Sys. 1997, 3, 251-264. [CrossRef]

21. Mawhen, J.; Willem, M. Critical Point Theory and Hamiltonian Systems; Springer: New York, NY, USA, 1989.

(C) 2020 by the authors. Licensee MDPI, Basel, Switzerland. This article is an open access article distributed under the terms and conditions of the Creative Commons Attribution (CC BY) license (http://creativecommons.org/licenses/by/4.0/). 